\title{
Google Docs: una alternativa de encuestas online
}

\author{
J. Jhonnel Alarco, Esmilsinia V. Álvarez-Andrade
}

Atrás han quedado los días en que las investigaciones se realizaban entre cuatro paredes de una institución. La investigación de hoy requiere un equipo de expertos que pueden estar ubicados en diferentes partes del mundo, sin el problema de las limitaciones geográficas [1]; por tanto, la colaboración es una parte vital del flujo de trabajo y la escritura no es la excepción.

Internet trajo una mejora notable en la forma de investigar, adoptando nuevas tecnologías, como el uso de herramientas de encuestas online, las cuales han revolucionado las antiguas formas de recogida de datos hasta el punto de que están quedando obsoletas. Sin embargo, esta apreciación no puede aplicarse a todas las formas de realización de encuestas, ya que existen parámetros que deben cumplirse.

Las encuestas online son una herramienta óptima para la recogida de información en aquellos entornos en los que la población de estudio analizada dispone de los requerimientos técnicos, la infraestructura y el nivel educativo y cognitivo necesarios para obtener resultados que puedan ser extrapolables y que limiten los efectos de los diferentes tipos de muestreo [2].

Esta clase de requerimientos se suele dar fundamentalmente en determinados tipos de organizaciones que se caracterizan por el hecho de que la mayoría de sus miembros tienen fácil acceso a entornos virtuales.

Un inconveniente es la poca tasa de respuesta, referida al porcentaje de individuos que finalizan el cuestionario del total de invitados a participar [3]. Entre las ventajas [3] de este tipo de encuestas, utilizando Internet para recoger información, podemos considerar las siguientes:

- Relativa facilidad de aplicación mediante la creación de cuestionarios complejos, en un mínimo tiempo, sin necesidad de tener conocimientos de programación de páginas web.

- Coste económico reducido por la eliminación de gastos de papelería, de envío y de administración, lo que favorece la posibilidad de realizar estudios de grandes muestras.

- Posibilidad de mejorar, variar y sofisticar las encuestas, desde comprobaciones de validez de las respuestas en tiempo real a la aleatorización de preguntas.

- Inserción de contenido multimedia, que haría más atractiva la encuesta, ventaja de la que carecen las encuestas de tipo tradicional.

- Posibilidad de enviar múltiples invitaciones y recordatorios cuando se produce, en un principio, poca respuesta al cuestionario online.

- Capacidad de almacenar automáticamente las respuestas, en una base de datos en formato electrónico, para su posterior interpretación.

- Eliminación de los errores generados por la introducción manual de respuestas.

En la web podemos encontrar numerosas aplicaciones que crean y comparten documentos de texto, hojas de cálculo y presentaciones; fundamentalmente son gratuitas, como Google Docs (https://docs. google.com), Microsoft Office Live (http://www. officelive.com), Zoho (http://www.zoho.com) y Ulteo (http://www.ulteo.com). Muchas otras son de pago.

De entre todas las aplicaciones presentadas, sólo Google Docs y Zoho permiten realizar formularios en línea; de ambas, sin duda Google Docs es la más conocida e intuitiva, y además está en castellano [4].

Google Docs es una interfaz disponible libremente para compartir, editar y dar seguimiento de documentos online; incluye un procesador de textos, una hoja de cálculo, un programa de presentación básica y otras funciones más.

Uno de los servicios más interesantes que ofrece Google Docs es la creación de formularios a partir de hojas de cálculo: permite de forma rápida y sencilla editar formularios destinados a la elaboración de encuestas para la creación de bases de datos que pueden orientarse a la realización de trabajos de investigación.
Facultad de Farmacia y Bioquímica (J.J. Alarco); Facultad de Medicina (J.J. Alarco, E.V. Álvarez-Andrade); Universidad Nacional San Luis Gonzaga; Ica, Perú.

Correspondencia: Dr. J. Jhonnel Alarco. Urb. Santa Rosa del Palmar, X-25. Ica, Perú.

E-mail:

jhonnelalarco@hotmail.com

(c) 2012 Educación Médica 
En la actualidad, la Facultad de Medicina Daniel Alcides Carrión, de la Universidad Nacional San Luis Gonzaga, en Ica (Perú), está utilizando este tipo de encuestas online con una amplia aceptación por parte de los docentes y estudiantes.

Estas herramientas, con el apoyo de las nuevas tecnologías, deben de ser ampliamente aceptadas por las comunidades universitarias procurando que sean gratuitas y sencillas en su aplicación. En este sentido, Google Docs cumple los requisitos básicos y ventajas de las encuestas online, por lo cual se debe promover y difundir su uso, tal como ocurre en otros países donde resulta habitual el empleo de este tipo de tecnologías.

\section{Bibliografía}

1. Phadtare A, Bahmani A, Shah A, Pietrobon R. Scientific writing: a randomized controlled trial comparing standard and on-line instruction. BMC Med Educ 2009; 9: 27.

2. Gras RM, Pérez MAM, Guardiola MCA. El uso de técnicas de investigación en línea: desde el análisis de logs hasta la encuesta electrónica. III Congreso de Metodología de Encuestas; Granada, 2004.

3. Braithwaite D, Emery J, De Lusignan S, Sutton S. Using the internet to conduct surveys of health professionals: a valid alternative? Fam Pract 2003; 20: 545-51.

4. Pastor JM. Los formularios en línea como herramienta telemática para interactuar con los estudiantes. @tic Revista d'Innovació Educativa 2009; 3: 79-83. 